\title{
Exploring the Ambulatory Transitional Care Experience from Residential Aged Care Facilities (RACF) to Ambulatory Care Services
}

\author{
Anthony Paul O’Brien, Michelle Giles, Lisa Corbett, Sushilla Wagener, Linda Ross, Kamana Bantawa, \\ Kerry Cooper, Darran Paul, Deborah Barnes and Karen McNeil
}

\begin{abstract}
Objective(s): To explore the transitional care journey through Ambulatory care Services (ACS) for older residents from Residential Aged Care Facilities (RACF). To develop a clearer understanding of older residents needs and any gaps in current services provided; and to inform the development of a model of care to improve the resident's transitional care journey.
\end{abstract}

Study Design: A qualitative project design using extensive stakeholder engagement

Method: The Ambulatory Care (AC) experience was explored through semi-structured interviews with residents and their carers to determine gaps in transitional care continuity. Focus groups with RACF and ACS staff were also utilized. Journey mapping was used to support anecdotal evidence.

Results: Three residents and 2 carers were interviewed and a total of 40 RACF and ACS staff attended 5 focus groups. Principal Findings: Qualitative data analysis identified four main themes across the transition journey:

- Inconsistent and adhoc communication;

- Just waiting around;

- Is it doing more harm than good?; and

- Unmet expectations.

Conclusion:

The results of this study have highlighted shortcomings in the provision of quality care in this transitional care group of older clients. A collaborative approach across organizational boundaries is necessary to ensure the development of an integrated person centered model to ensure the best transition to ambulatory care for RACF residents exists.

Keywords- Qualitative research; ambulatory care; outpatient clinics; Residential Aged Care; transitional care

\section{INTRODUCTION}

There have been many studies on transitional care for older people but predominantly they have been conducted in Emergency Departments (EDs) and inpatient settings where basic care needs for this vulnerable group are provided [1, 2]. In the Ambulatory care (outpatient) context it is known that good transitional care ensures coordination and continuity of healthcare and reduces the risk of adverse consequences. There is a shortage of literature however that focuses specifically on the transitional aged care journey.

The Ambulatory Care Services (ACS) referred to in this study are part of a large 600 plus bed tertiary referral hospital offering a range of medical and surgical specialist, nursing and allied health services in an outpatient setting [3]. Residential Aged Care Facility (RACF) residents can be routinely referred to the ACS after a hospital admission, with anecdotal evidence suggesting visits can be fragmented, time consuming and stressful. In some cases residents can spend hours unescorted in the ambulatory care environment with no medication, nutrition or familial support.

In the lead up to this study, ACS nurses conducted a local audit of older people from residential care attending the ACS identifying that many have complex chronic illnesses, mobility problems, incontinence, pressure injury and skin integrity problems and/or cognitive impairment. The audit also identified that clinics can often run late and waiting for ambulance transport back to residential care can be fraught with delays.

Extended and unsupervised wait times do not suit frail older people who often have complex chronic illnesses and cognitive impairment [4, 5] requiring supervision. Because of their age and level of acuity generally, a visit to ambulatory care in the hospital can place the older person at risk of delirium associated with the absence of prescribed medications, dehydration and nutrition deficit. The older person with mobility, 
dehydration and nutrition problems is also at an increased risk of developing a pressure injury [6].

This paper discusses the findings associated with a study aimed at exploring the transitional ambulatory care journey for residential care to ambulatory care.

\section{LITERATURE REVIEW}

Over the next forty years in Australia the number of 65 to 84 year olds is expected to more than double, with the number of people living beyond 85 years of age projected to quadruple [7]. Approximately 169,000 frail and disabled older people are cared for in residential care in Australia, with over 76 per cent of these residents assessed as having high levels of dependency related to activities of daily living, behavioral diagnoses and/or other complex health care needs [8]. More than 75 per cent of RACF residents have been diagnosed with a mental illness, with over half suffering from dementia [8] Many residents fall into the high level of care category, measured by the Aged Care Assessment Funding Instrument (ACFI) and they are often in need of an acute care stay in hospital, or require an ambulatory care appointment [8].

Transitional care is defined as a set of actions designed to ensure the coordination and continuity of health care as patients transfer between different locations, or different levels of care within the same location [9]. The literature is sparse on the unique challenges associated with residential care patients attending ambulatory care or outpatient clinics; however the local clinical experience is that many older residents attend unescorted, or with a relative or carer, who is often an older person themselves.

Because older patients are more likely to suffer from multiple comorbidities, including functional impairments and dementia they are more likely to receive care from a number of specialist providers [10]. This patient cohort are more frequently in transition between healthcare settings [9] which places them at greater risk of adverse health outcomes resulting from inadequate transfer of critical treatment information [10]. There is thus an urgent need to streamline Ambulatory Care (AC) transitions in tertiary referral hospitals [7].

Good transitional care involves the careful coordination and continuity of healthcare agencies and people to reduce the risk of adverse consequences [9]. Researchers have explored the transitions between residential aged care facilities (RACFs) and hospital settings but the literature is silent on the experiences of residential care residents attending Ambulatory Care Services (ACS), or other similarly named hospital outpatient clinics. Patients in the AC setting are not admitted to hospital and can generally 'walk in and walk out', or be 'wheeled in and wheeled out' of the hospital [11].

Ambulatory care can also be provided in day surgeries [12], community-based health centers [13] and primary care settings; such as general practice medical centers in addition to AC [14]. Additionally, in Australia there is a strong agenda to expand ambulatory and community based health care delivery with the view of reducing the increase in emergency department and hospital admissions due to the growth of our ageing population [15].

Researchers from other countries report that the majority of nursing home residents require assistance with bathing, dressing, toileting and almost half require help with eating (Jones, 2002, as cited in [16]).

Given such high levels of dependency and acuity, transitions to other health care settings increase residents' vulnerability in transitional care [17]. Additionally, health care providers can operate in isolation and practitioners involved in the transition transfer are often unaware of the capacity of the receiving setting to deliver care [18].

During transition between health care settings there is a further increased risk to the patient due to errors in communication, inadequate transfer of information plus the involvement of multiple health professionals, family members and caregivers [10, 12, 19]. Transfers between residential care and the acute sector involve clinical handovers between residential care staff, ambulance officers and hospital staff [20] with a range of research literature suggesting that most patients transferring from nursing homes do so without vital information such as medication status, or level of cognitive impairment [10].

This communication process is further challenged in the case of residents from diverse cultural and linguistic backgrounds $[8,11]$. Furthermore, miscommunication of critical health information is more likely when the patients themselves are suffering from cognitive impairment or memory issues [17] or are not accompanied by an advocate to ensure that they receive effective care [10].

In the state of Victoria a group of providers adopted an envelope system to transfer documentation plus a checklist with critical clinical and handover information [20]. Alternatives to this process could be confidential web-based medical records or "smart-cards" that are carried by the patient [9].

\section{Local context}

The ACS outpatient clinic is an extremely busy service set in a large tertiary referral hospital in New South Wales (NSW) Australia. There are over 100,000 outpatient referral visits annually and in a six month period November 2012 to April 2013 25\% of clients referred were 70 years of age or over. Information was 
not available in the Patient Management System (PMS) to identify the clients residing in residential care at the time they attended the outpatient clinic appointment. Relying on national figures with an estimated $7.3 \%$ of people over the age of 70 years residing in Residential Aged Care Facilities (RACF) [21], potentially 1800 residents may be referred to attend ACS in a 12 month period.

\section{THE STUDY}

\section{A. Study aim}

The aim of the study was to explore the Ambulatory Care journey of RACF residents and to develop a clearer understanding of their needs and gaps in current services provided. This was achieved by:

- Exploring residents experiences of the ACS transitional journey

- Identifying RACF staff perspectives and concerns

- Identifying ACS staff perspectives and concerns

- Mapping several residents' transitional journeys to ACS.

This process informs the development of a model of care that will improve the resident's overall journey and outcome.

\section{B. Ethical approval}

Humans Research Ethics approval was granted at a District level through the Hunter New England Research Ethics Committee. Interview and focus group participants were provided with details of the purpose of the study prior to participation, ensuring informed consent. They were assured that their privacy would be protected at all times. Digital audio files of interviews and focus groups were stored in a password-protected file and pseudonyms and codes in reporting responses maintained confidentiality [22].

\section{METHOD}

\section{A. Design}

The study used qualitative triangulated methods of data collection through interviews and focus groups. This method was chosen to enhance description and provide explanation to gain a more complete understanding [23].

\section{B. Data collection}

Nursing Staff responsible for caring for residents in RACF's and ACS nursing, allied health and administrative staff were provided with information and invited via email and at ward meetings to participate in focus groups. Focus groups attempted to explore staffs' perceptions of the journey, identify resident needs and how well these needs were met, as well as highlight key issues, problems and gaps in service delivery, including opportunities for improvement.

Residents who had recently attended the ACS outpatient clinics were identified by RACF managers and provided with information about the study and the consent form. Three interviews with consenting residents across two aged care facilities and two carer interviews were conducted.

Interview questions followed the following format:

- Can you tell us about your experience of attending the outpatient clinic

- What did you find helpful

- What did you find unhelpful

- What would you like to see done differently.

- A list of prompts was used to encourage conversation.

Several patient journeys to ACS outpatient clinics were followed using specially developed audits tools to identify waiting times, transport details across the transition of care journey and to substantiate the anecdotal evidence.

\section{RACF staff focus groups}

Two focus groups were held with staff from two large residential care facilities and were conducted at their facility. A total of 21 staff attended the two focus groups. Registered Nurses (RNs), Enrolled Nurses (ENs), and Assistants in Nursing (AINs), Personal Care Assistants (PCA) and nursing managers were participants.

\section{Ambulatory care staff focus groups}

Three focus groups were conducted with ACS staff, one with administrative staff $(n=5)$ and the remaining two focus groups included clinical staff. A total of 14 RNs, Physiotherapists and ENs participated.

\section{Resident / Carer Interviews}

Three residents who had recently attended the ACS outpatient clinic consented to a face to face semi structured interview at their RACF. In two of these interviews the relative (carer) who escorted the resident to the outpatient clinic appointment also participated $(n=5)$.

\section{Data Analysis}

Qualitative data from focus groups and interviews were digitally recorded with the expressed written consent of participants and then transcribed verbatim. Transcripts were analyzed through open, axial and substantive coding [24] by individual researchers in the research team and 
then corroborated across researchers during scheduled coding sessions organized in the ambulatory care setting. Themes were derived using an inductive iterative and comparative process. Themes were finalized through the development of relationships and patterns in the transcribed data and agreement from the clinical staff in the research group [25].

\section{RESULTS}

\section{A. Resident Journey Mapping}

Several resident ambulatory care journeys were mapped. The following describes just one example. A stretcher bound female resident arrived via ambulance transport at 2pm for an appointment, ambulance transport staff placed her in the care of ambulatory care staff. The resident required an $\mathrm{x}$-ray before her appointment and was seen shortly after her return to the outpatient clinic. However the resident was not transported back to her residential facility until 2am the next morning by ambulance transport. Furthermore, no documentation had been sent with the patient from the RACF, she had attended the outpatient clinic unescorted and during the 12 hour ordeal endured 2 unit transfers as the clinic and transit wards closed at end of business. A follow-up phone call from concerned ambulatory care staff the following day revealed that the resident had been extremely upset and anxious on her return to her facility and that staff had noted increased confusion.

\section{B. Qualitative themes from Focus groups and interviews}

Predictably, the diverse groups participating in focus groups and interviews highlighted different aspects of concern in the transition of care for the residential care resident. There were four predominant themes across the qualitative data, as follows:

\section{Theme 1: Inconsistent and adhoc communication}

The most prominent concerns were echoed in the theme regarding communication shortfalls, especially reflecting the lack of information provided and inconsistent approach to providing necessary patient information at the right time for every patient across the transitional journey. These problems occurred between facilities and even within facilities. Participants reported that the adhoc and inconsistent approach to communication appears to be systemic, occurring across all services involved in the transitional journey.

Examples given in the focus groups related to ambulatory care failing to inform the residential care facility of cancelled or rescheduled appointments; ambulance services not communicating transport cancellations or delays; ACS not receiving the residents past and current medical history, or the outcome of clinic appointments not being communicated back to the residential care staff. There were instances where ambulatory care had cancelled clinic appointments but the residential care place was not informed of the cancellation. Residents had been transported to ambulatory care only to find their appointments cancelled or rescheduled.

A breakdown in communication was highlighted between the residential care place and the ambulance transport system. In the majority of instances for example ambulance transport is required to transport residents to and from their appointments, so there are many challenges experienced through a lack of communication between the two services. This has led to residents being late for appointments, or even missing them completely.

\section{An RACF staff member shared their experience:}

"We can book patient transport 2 weeks in advance and come the day, you ring up and they say, oh no we don't have that booking, or you know, they just don't turn up, no phone call to say we won't be able to make it today, can we make it another day..." (RACF staff, FG1)

There appears to be agreement from both residential care and ambulatory care participants that the provision of information between services is inconsistent and adhoc and that the problem is bi-directional. What was apparent is that there is no standard documentation process in place. Even when information is sent with the resident, this is not always handed on by ambulance transport officers to ambulatory care staff, and vice versa. The problem is further compounded when residents are either accompanied by a family member, an escort who does not know the residents' history, or the resident is not accompanied by an escort at all.

Residential care staff expressed frustration at not being able to contact anyone related to the clinic visit who might be able to provide any information after the appointment, and that no follow-up information is provided from the ambulatory care clinic.

\section{A residential care staff participant explains:}

"She came home with a sling on her arm but how long do they want us to have it on -maybe two weeks? With having this experience I have tried to ring to speak to someone in the clinic and it's frustrating for both parties..." (RACF staff, FG2)

Concerns were shared by the ambulatory care staff participants who believe they could better care for residents if they had more information provided. There was difficulty in the first instance in identifying clients as RACF residents. The current Patient Management System (PMS) does not enable clients to be flagged as coming from a residential care facility. The ambulatory 
care staff participants believed that if they had advance warning it would assist them in providing a better patient experience for the resident.

One ambulatory care staff member suggested:

“There doesn't seem to be enough information on the day's clinics if a patient is coming from an aged care facility, so I think we probably need a little bit more information on the day - usually we don't know that the patient is coming from an aged care facility until they have actually arrived with the ambulance and then everything mushrooms out and chaos happens." (ACS staff, FG3)

Ambulatory care nursing staff found it difficult to care for residents when they have little background information regarding medications, dietary restrictions and cognitive and mobility levels. Sometimes residents are accompanied by an escort or a family member, however in many instances the person assisting the resident is not familiar with their medical history, or they are not accompanied by an escort at all.

\section{Another ACS nursing staff participant added:}

"I think one of the key issues is when a patient comes in with transport and they are on a stretcher and they are elderly...they often don't have a carer with them and we don't know what is wrong with them. We don't know whether they are diabetic, we don't know whether they have got dementia. We don't know whether they can mobilize. You know we have got nothing." (ACS staff, FG3)

There was a general consensus between ambulatory care and residential care nursing staff focus group participants that the communication between the ambulance transport service, residential care and ambulatory care needs significant redesign and improvement to provide a safer and more comfortable experience for the residents.

\section{Theme 2: Just waiting around}

Residents, carers, residential care and ambulatory care nursing and administrative staff spoke of major concerns related to patients waiting and inconsistent and unreliable transport to and from appointments. Unreliable transport led to long delays where patients from residential care were required to wait. This was disruptive to their daily routines, raised their anxiety levels and increased the risk of adverse events. In some cases residential care staff came in on their days off and used their own cars to transport residents to an appointment to offset problems with waits and unreliable transport.
Ambulance transport is the most common mode of transport for residential care patients. The main challenges highlighted by staff from residential care were the unreliability of ambulance transport, sometimes arriving very late, or not at all, and extensive delays while the resident is waiting to be returned back to the facility. For ambulatory care staff participants a major concern highlighted was the frequent time delays associated with waiting for ambulance transport to return the resident to the RACF. The transport of the resident is classified as a category 5 (a low priority classification) so there are often long delays.

A residential care staff participant said:

"...they have to sit there then to wait for an ambulance and sometimes they can be getting back here [RACF 3 at $11 \mathrm{pm}$ at night and they have sat there since their appointment, missed their dinner, missed their medications, missed everything distressed and tired." (RACF staff FG1)

Again the main issue highlighted by resident and carer interview participants was the wait and being late for appointments.

As one resident who regularly visits the outpatient services stated:

"Last appointment I waited 3 hours on my own with no food or drink. Ambulance took me for my appointment, took me to clinic and booked me in at the reception desk...Some ambulances are able to wait if I get straight in for my appointment /..../ I don't like going home after dark." (Resident, Interview 2).

The waiting time related to delayed and unreliable transport to appointments also impacts on family members and carers who on occasion have arranged in advance to meet their elderly relative in ACS. When transport is delayed this causes anxiety for family members who are waiting for their elderly loved one where they do not know if they have arrived to the ACS, or if they have been seen and or left already.

\section{Theme 3: Is it doing more harm than good}

The ambulatory care and residential care staff participants expressed concern about the distress and anxiety caused to residents and their escorts/carers when waiting for long periods of time in the clinic. The long wait for transport, crowded waiting areas with no real facilities to cater to the needs of residents requiring assistance, no ability to maintain privacy, unfamiliar surroundings and the fear of being lost in the hospital, make the ambulatory care clinic visit very daunting for many elderly residents. 
A residential care staff member explains the impact a clinic visit can have on an elderly resident:

"...old people live and breathe by the doctor and the clock so they are thinking about if they are going to miss their appointment and they're going to make the doctor cross, and they're anxious anyway and they wouldn't have drank much for breakfast because they know they're going out and you don't want to be going to the toilet because you don't know if you're going to be able to find a toilet and so the beginning of their dehydration has already started as it is.

...they come back and are exhausted and tired and some of them have been crying because they have been sitting there waiting." (RACF staff FG2)

Residential care staff described instances where residents returned from their visits in sub optimal conditions.

"..yeh sometimes they [residents] come back with pressure sores, they come back with bruises on their arms, yeh like really bad, or they have had their arm hit, or something like that and then you've got a skin tear and you've got to fix it." (RACF staff FG2)

Another residential care staff member says that when residents return to the facility:

"I have found that they [residents] are often incontinent, in wet clothes or wet pads and they have been sitting in it all day because they haven't had anyone look at them." (RACF staff FG1)

On the other hand, ACS staff are aware of long waits in their service for some residents and the lack of facilities within their department to provide adequately for the resident during this time. This is particularly the case if the resident has limited mobility, or is stretcher bound. The staff describe instances where the stretcher bound resident may be left in a corridor alcove with no privacy available (no curtains) if requiring toileting, or other care, and no escort. Staff may also be required to spend considerable time organizing transfer of the resident to the Transit Lounge, or even a hospital ward if ambulance transport is not available prior to the Transit Lounge closure at $6 \mathrm{pm}$.

Both residential care and ambulatory care staff question the need in many cases for referrals and outpatient visits in the first instance and believe that many residents are referred routinely as part of the generic discharge process. They believe that if each case was considered individually and if clinicians were aware of some of the difficulties faced when a resident visits ACS, they would not refer but perhaps arrange follow-up alternatives.

\section{Theme 4: Unmet expectations}

There are certain expectations that each of the stakeholder groups had about what each facility/service could provide across the transitional experience and the themes from the focus groups clarify that many of these expectations are not being met. This is compounded by the need to improve the communication and connectedness between relevant services.

There is an expectation from residential care staff that residents will be seen promptly and that ambulance transport officers will accompany the resident to the clinic, then return them back to the RACF immediately, or within a reasonable and acceptable period. Conversely, ambulatory care staff have expectations that documentation will accompany the resident and that the RACF will have arranged adequate medications, food, fluids and supervision in advance to provide for the resident's needs, particularly if there is an unforeseen delay.

It is acknowledged by all participants that the facilities provided in ambulatory care and the transit lounge where many residents can spend several hours are not always adequate to fully cater to their needs. Staff participants noted that the environment is crowded and can appear chaotic which can "overwhelm" residents, particularly if they are by themselves. The facilities provided in the ACS environment fall short of what is required to provide adequate care for elderly residents over prolonged periods of time.

One residential care staff member when referring to her experiences when escorting residents to ACS said:

"They need a suitable facility or area for managing demented patients who may need a shower, toileting or general hygiene to allow for personal care with privacy and dignity." (RACF staff FG2)

Key areas of concern highlighted by the residents and their carers, as well as residential staff participants, were the problems navigating the hospital building and car parking. Simple things like finding a toilet or the appropriate outpatient clinic can be difficult. The lack of proper signage and use of health terminology to describe units and departments can make it difficult for visitors to follow directions.

An ambulatory care clerical staff participant stated:

"You find that quite often they are scared. Because they are disorientated and then they don't know where they are and there is not enough signage around the place for people to actually get to clinics...they get lost all of the time." (ACS clerical staff FG5)

From a local context parking was mentioned frequently by carers and staff who escort residents and this was mainly about the lack of disabled parking 
facilities to fulfil the needs of elderly residents, carers or escorts, especially when the client was cognitively impaired or had mobility problems. There was a view that although disabled parking is available, access to these spaces is challenging especially when wheelchairs are not readily available within the hospital premises for less mobile people.

A carer said:

“... even though there is disabled parking in the big car park across the road, they have to walk up stairs or up the hill to get to the hospital. That is not disabled parking, you know it is just totally inadequate and it should be addressed." (Carer interview 1).

Residential care staff also echoed the same sentiments in terms of inadequate parking facilities. Those staff who do escort residents outside of their own working hours said that it requires two of them to escort because of the difficulty parking and the inadequacy of disabled parking.

"Start with the parking, yep that is very difficult at [this facility], well it took two of us, you can't go on your own you can't just ask the person to sit and wait till I park. You have to go with two escorts...well, you wouldn't be able to leave them anyway because you can't get the resident out and sit them somewhere in case they're not there when you come back." (RACF staff FG1)

\section{Model of Care developed}

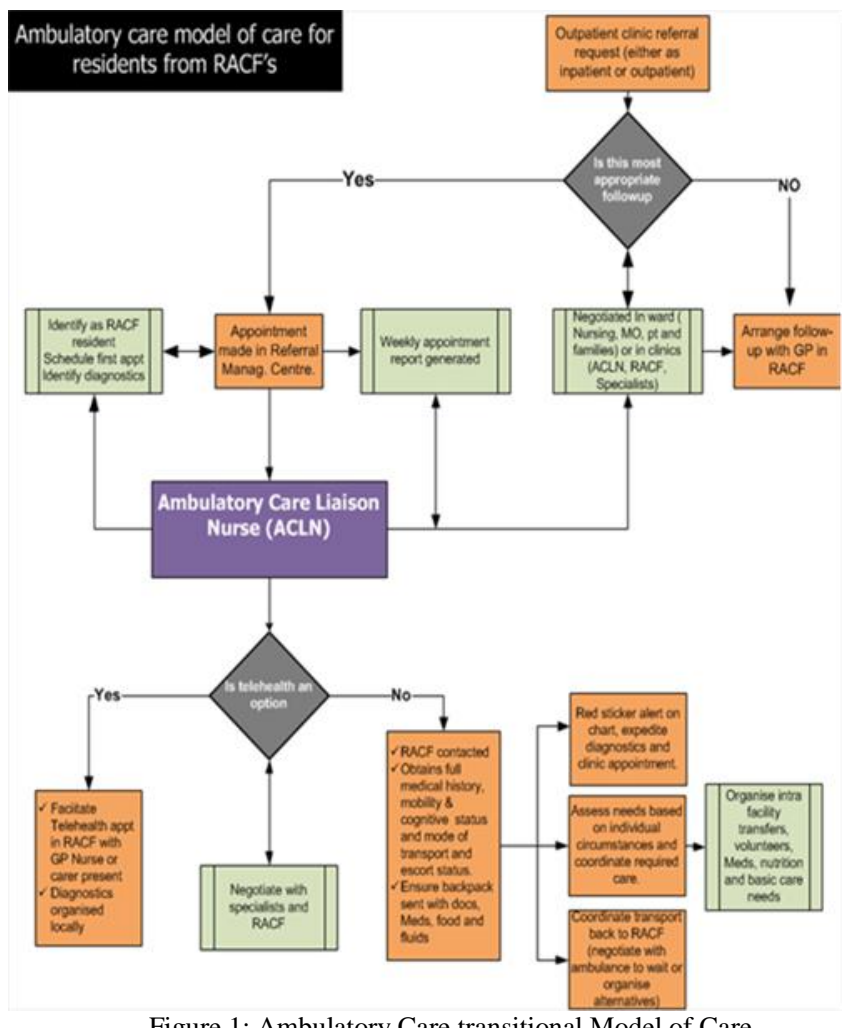

Figure 1: Ambulatory Care transitional Model of Care
This model of care (Fig.1) was developed based on the qualitative results presented above. The mapping process identified four main touchpoints of the ambulatory care journey; making the referral, making the appointment, transport and attending the appointment. A content and thematic analysis highlighted issues of concern as outlined in detail in the themes. From here the team identified actions to address these issues and developed a model of care around these actions across the journey touchpoints. The model hinges on a central pivotal role of Ambulatory Care Liaison Nurse (ACLN) that would eliminate much of the bidirectional poor communication that currently exists. The model also highlights major 'touch points' across the journey from referral of residents to outpatient clinics, to transport back to RACF's where further work needs to be done to streamline and integrate services and processes.

Alternative and more appropriate methods of followup are also considered in the model as well as tele health options which are currently emerging in local outpatient clinic settings.

\section{DISCUSSION}

There is no doubt that elderly residents residing in RACFs are our most vulnerable population group; many have complex chronic illnesses, mobility problems, incontinence, pressure injury and skin integrity problems and/or cognitive impairment. For elderly residents who are taken out of a familiar environment and who do not have their basic needs met, the risk of adverse events occurring is high and can cause stress and anxiety increasing the need for risk management [9].

There have been many studies on transitional care for older people predominantly focusing on Emergency Departments (EDs) and inpatient units [1, 2] but the transitional journey for residents from RACF's attending ambulatory care has virtually been ignored. The results of this study explore previously unchartered territory and provide foundational discovery work to inform transitional care models. With an increased focus on provision of health care services away from traditional ED and inpatient settings [26] it is timely to closely examine current practices and implement an integrated approach aimed at providing a safe and high quality transitional health care journey.

It has been well established that good transitional care ensures co-ordination and continuity of healthcare and reduces the risk of adverse consequences [9]. The results of this quality project have highlighted that there are major gaps in ambulatory care transitional service delivery across RACFs and in the context of ambulance transfers that can lead to harmful consequences for elderly residents. The problems highlighted in this study appear to be systemic failures across a broad spectrum of 
the health care continuum ranging from ritualistic referral processes, inadequate discharge planning and lack of a person centered approach to follow-up. This is compounded by service providers choosing to work in isolation with poor communication between and within services.

There is strong consensus across services that a central point of contact within ambulatory care would be valuable and improve bi-directional communication to improve patient care. A clinician (often a nurse) who is able to bridge the different health care settings can assist in the transition by coordinating care, reconciling medication information and being the key contact for patients, family, caregivers and other health practitioners $[9,27,28]$. Such a person could be contacted in advance by residential care staff, information about the resident could be given over the phone and this person could also receive the resident on arrival. Despite long wait times frequently occurring there appears to be little effort put into preparing the resident if this occurs. The project team have developed a model of care based on the above concept (Figure 1) with an ambulatory care liaison nurse to coordinate the referral and transition process. This model will be implemented and evaluated when funding is sourced.

A key component of this model is that alternative methods of follow-up should be considered by medical staff for this group of patients. Currently routine referral to outpatient clinics occurs in many instances and other forms of follow-up counteract the detrimental impact on residential care residents who currently attend. Alternative methods to be considered are follow-up with local General Practitioners, Nurse Practitioners and Allied Health Staff within residential care facilities and better use of technology and telehealth options, such as video conferencing between service providers. In the $\mathrm{ED}$, it has been recommended that alternative waiting processes could be adopted for older patients [29, 30] and this could be applied in outpatient clinics. There are examples where outpatient clinics have been conducted in nursing homes to reduce problems with transition and communication of medical information [31].

Stakeholder engagement is a vital next step in the development of a model of care that addresses the shortcomings of all service providers and with a holistic approach to model development, focusing on improved communication systems between services, maintenance of hydration and nutrition, continence management, mobility amelioration and medication administration. Any successful practice change however must facilitate 'buy-in' from each of the organizational stakeholders [32].

Future predictions related to our ageing population and a push nationally to provide alternative methods of service delivery away from acute care facilities will see rapid growth in ambulatory care [15]. It is imperative that the issues highlighted in the results of this study be addressed to ensure a safe and sustainable model of care is developed and evaluated for this group of elderly residents and patients attending ambulatory care services.

The limitations of this study do not outweigh the importance of the findings. A larger sample of residents and carers would have provided a deeper consumer perspective. Interviewing a sample of Ambulance workers would have provided another perspective. The issues involved between residential care settings sending clients to the outpatient departments that do not meet their expectations in terms of timely service delivery must be taken in the context of a very busy Ambulatory care center undergoing redesign.

\section{CONCLUSION}

There are shortcomings highlighted in the current service model for residential care residents attending ambulatory care in hospitals globally. Stakeholder engagement and a holistic approach to model development focusing on improved communication systems between services and an integrated approach to provision of care across the care continuum is a vital next step to improve the quality and safety care for the older .outpatient. A great deal has been gained from this study which has combined different service users and consumers and novice nurse and allied health staff researchers in a research project yielding greater clarity around the Transitional care journey.

\section{ACKNOWLEDGMENT}

The project was initially funded by a Nursing and Midwifery Innovation scholarship provided by the Nurses and Midwives Office of the NSW Ministry of Health.

The authors would like to thank all the clinicians who participated in focus groups for their valuable contribution.

We would also like to thank Ms Wendy Pudney, who was involved in the initial phase of the project, for her sponsorship and support to the project as an Ambulatory Care Manager of the project at the time.

\section{REFERENCES}

[1] G. Arendts and K. Howard, "The interface between residential aged care and the emergency department: A systematic review," Age and Ageing, vol. 39, pp. 306-312, 2010.

[2] J. C. Finn, L. Flicker, E. Mackenzie, I. G. Jacobs, D. M Fatovich, S. Drummond, et al., "Interface between residential aged care facilities and a teaching hospital emergency 
department in Western Australia," Medical Journal of Australia, vol. 184, pp. 432-435, 2006.

[3] NSW Health, "Clinical Services Directory - Ambulatory Care Services," 2011

[4] M. E. Exum, B. J. Phelps, K. E. Nabers, and J. G. Osborne, "Sundown syndrome: Is it reflected in the use of PRN medications for nursing home residents?," Gerontologist, vol. 33, 1993.

[5] J. Cohen-Mansfield, D. Garfinkel, and S. Lipson, "Melatonin for treatment of sundowning in elderly persons with dementia a preliminary study," Archives of Gerontology and Geriatrics, vol. 31, pp. 65-76, 2000.

[6] Clinical Excellence Commission, "Pressure Injury Prevention and Management," Clinical/ Patient Services - Governance and Service Delivery, Ed., ed. Available at http://www.cec.health.nsw.gov.au/programs/pressure-injuryprevention-project, 2014

[7] Commonwealth of Australia, "Australia to 2050: Future challenges," Attorney General's Department, Ed., ed. Canberra: CanPrint Communications Pty Ltd, 2010.

[8] Australian Institute of Health and Welfare, "Residential aged care in Australia 2010-11: A statistical overview," Australian Institute of Health and Welfare, Ed., ed. Canberra: AIHW, 2012.

[9] E. A. Coleman, "Falling through the cracks: Challenges and opportunities for improving transitional care for persons with continuous complex care needs," Journal of the American Geriatrics Society, vol. 51, pp. 549-555, 2003.

[10] C. Kessler, M. C. Williams, J. N. Moustoukas, and C. Pappas, "Transitions of care for the geriatric patient in the emergency department," Clinics in Geriatric Medicine, vol. 29, pp. 49-69, Feb 2013.

[11] Productivity Commission, "Caring for Older Australians: Overview, Report No. 53: Final Inquiry Report," ed. Canberra, 2011.

[12] J. M. Nelson and J. M. Carrington, "Transitioning the Older Adult in the Ambulatory Care Setting," Association of periOperative Registered Nurses, vol. 94, pp. 348-361, 2011.

[13] P. Bywood, E. Jackson-Bowers, and S. Muecke, "Initiatives to integrate primary and acute health care, including ambulatory care services: PHC RIS Policy Issue Review," Primary Health Care Research \& Information Service, Ed., ed. Adelaide: Available http://www.phcris.org.au/phplib/filedownload.php?file=/elib/li b/downloaded_files/publications/pdfs/phcris_pub_8357.pdf, 2011.

[14] T. K. Gandhi, S. N. Weingart, J. Borus, A. C. Seger, J. Peterson, E. Burdick, et al., "Adverse drug events in ambulatory care," New England Journal of Medicine, vol. 348, pp. 1556-1564, 2003

[15] Health Reform Implementation Taskforce, "Ambulatory and community-based care: A framework for non-inpatient care," Department of health, Ed., ed: Government of WA, 2007.

[16] L. L. Popejoy, "Hospital discharge destination decisions: Exploring congruence in frail elders, their family members and health care teams' decisions," Doctor of Philosophy, Faculty of the Graduate School, University of Missouri-Columbia, 2007.

[17] M. A. LaMantia, L. P. Scheunemann, A. J. Viera, J. BusbyWhitehead, and L. C. Hanson, "Interventions to improve transitional care between nursing homes and hospitals: A systematic review," Journal of the American Geriatrics Society, vol. 58, pp. 777-782, 2010

[18] E. A. Coleman and R. A. Berenson, "Lost in transition: Challenges and opportunities for improving the quality of transitional care," Annals of Internal Medicine, vol. 141, pp. 533-536, 2004

[19] C. Moore, J. Wisnivesky, S. Williams, and T. McGinn, "Medical errors related to discontinuity of care from an inpatient to an outpatient setting," Journal of General Internal Medicine, vol. 18, pp. 646-651, 2003.

[20] M. K. Belfrage, C. Chiminello, D. Cooper, and S. Douglas, "Pushing the envelope: Clinical handover from the aged-care home to the emergency department," The Medical Journal of Australia, vol. 190, pp. S117-S120, 2009.

[21] S. Gadzhanova and R. Reed, "Medical services provided by general practitioners in residential aged-care facilities in Australia," Medical Journal of Australia, vol. 187, pp. 92-94, 2007.

[22] National Health and Medical Research Council, "Nationa Statement on Ethical Conduct in Human Research," ed, 2007.

[23] J. Morse and L. Niehaus, Mixed method design: principles and procedures. California: Left Coast Press Inc, 2009.

[24] B. G. Glaser and A. L. Strauss, The Discovery of Grounded Theory strategies for qualitative research. Chicago: Aldine Transaction, 1997.

[25] S. Thorne, L. Jensen, M. H. Kearney, G. Noblit, and M. Sandelowski, "Qualitative metasynthesis: Reflections on methodological orientation and ideological agenda," Qualitative Health Research, vol. 14, pp. 1342-1365, 2004.

[26] Victorian Government Department of Human Services, "The Victorian ambulatory care sensitive conditions study: Preliminary analyses," ed, 2001.

[27] B. A. Swan and S. A. Haas, "Health care reform: Curren updates and future initiatives for ambulatory care nursing," Nursing Econmic\$, vol. 29, pp. 331-334, 2011.

[28] M. D. Naylor, D. Brooten, R. Campbell, B. S. Jacobsen, M. D Mezey, M. V. Pauly, et al., "Comprehensive discharge planning and home follow-up of hospitalized elders: A randomized clinical trial," The Journal of American Medical Association, vol. 281, pp. 613-620, 1999.

[29] M. R. Nolan, "Older patients in the emergency department: what are the risks?," Journal of Gerontological Nursing, vol. 35, pp. 14-8, Dec 2009.

[30] J. Considine, R. Smith, K. Hill, T. Weiland, J. Gannon, C. Behm, et al., "Older peoples' experience of accessing emergency care," Australasian Emergency Nursing Journal, vol. 13, pp. 61-69, 2010

[31] S. N. Nabi and W. Nabi, "Should clinics be held in homes?," in Nursing \& Residential Care vol. 7, ed, 2005, pp. 136-136.

[32] T. A. Cortes, S. Wexler, and J. J. Fitzpatrick, "The transition of elderly patients between hospitals and nursing homes: Improving nurse-to-nurse communication," Journal of Gerontological Nursing, vol. 30, pp. 10-5, Jun 20042004. 


\section{AUTHOR'S PROFILE}

Prof. Anthony (Tony) Paul O'Brien, is the Head of Discipline Nursing, University of Newcastle, Australia. He held the position of the Senior Clinical Lead Research at the Nursing and Midwifery Research Centre (NMRC), Hunter New England Local Health District. Professor O'Brien holds degrees in Bachelor of Arts - Conferred September 22nd 1989 (Macquarie University, Australia) Education and Politics majors; Master of Educational Studies - Conferred May 71994 (The University of Newcastle, Australia) and Doctor of Philosophy - Conferred 31 March 2001 (University of New England, Australia).

Mrs Michelle Giles is a Clinical Nurse Consultant for Research and Knowledge management in the Nursing and Midwifery Research Centre (NMRC), Hunter New England Local Health District Health, Australia. Her main role is supporting clinicians across disciplines to work in partnership to achieve their research goals.

Ms Lisa Corbett is a Registered Nurse in Ambulatory Care Services, Royal Newcastle Centre, Hunter New England Local Health District, Australia.

Ms Sushilla Waganer is Acting Operation manager, Ambulatory Care Service, Royal Newcastle Centre, Hunter New England Local Health District, Australia.

Ms Linda Ross is an Orthopaedic Clinical Nurse Consultant at Royal Newcastle Centre - Bone and Joint Institute, Hunter New England Local Health District, Australia.

Ms Kamana Bantawa is a Research Officer in the Nursing and Midwifery Research Centre (NMRC), Hunter New England Local Health District, Australia.

Ms Kerry Cooper held the position of Clinical Nurse Consultant, Rheumatology at John Hunter Hospital, Hunter New England Local Health District, Australia.

Mr Darran Paul is a Facility Manager at Quakers Hill Nursing Home, Australia.

Ms Deborah Barnes is a Clinical Nurse Specialist in the Ambulatory Care Serves, Royal Newcastle Centre, Hunter New England Local Health District, Australia.

Ms Karen McNeil is a Research Officer at Nursing and Midwifery Nursing and Midwifery Research Centre (NMRC), Hunter New England Local Health District, Australia. 